\title{
Technology of the Local Cross-linking (Part 1): Keratotensotopography and Vacuum-compression Topographic Test-New Diagnostic Possibilities for Studying the Local Biomechanical Properties of Cornea
}

\author{
${ }^{1}$ SI Anisimov, ${ }^{2}$ SYAnisimova, ${ }^{3}$ AS Mistryukov, ${ }^{4}$ NS Anisimova
}

\begin{abstract}
Aim: The aim of this study is the development and evaluation of clinical significance methodology for determining mechanical stress (MS) and elasticity of the cornea comparable to its topographic settings.

Materials and methods: Combining the data of optical pachymeter, keratotopography, and pneumotonometry through our special software allows us to get Lokolink MS distribution in the cornea in the form of maps, which we propose to call keratotensotopogram (KTT).

Results: Normal KTT has the form of concentric, uniform, and symmetrical zones. In keratoconus (KC), the corneal center is more tense than peripheral part of cornea. The focus zone of $\mathrm{KC}$ may coincide with the region of maximum MS or may be out of this area. In the process of vacuum-compression test, the reaction of profile of the cornea was markedly different in patients with healthy corneas and in patients with $\mathrm{KC}$. In all cases of healthy cornea, the curvature increased in the center. In KC, the cornea became more flat in the center under high intraocular pressure (in $100 \%$ of cases).
\end{abstract}

Conclusion: The KTT ineyes with the cornea without pathology differs from KTT in the different cases of KC. The KTT can be used for the diagnosis and prediction of cornea's state to perform the plan of UV irradiation areas for corneal collagen cross-linking.

Keywords: Corneal collagen cross-linking, Corneal topography, Keratotensotopogram, Vacuum-compression test.

How to cite this article: Anisimov SI, Anisimova SY, Mistryukov AS, Anisimova NS. Technology of the Local Cross-linking (Part 1): Keratotensotopography and Vacuumcompression Topographic Test-New Diagnostic Possibilities for Studying the Local Biomechanical Properties of Cornea. Int J Kerat Ect Cor Dis 2017;6(1):14-16.

Source of support: Nil

Conflict of interest: None

${ }^{1}$ Professor, ${ }^{2}$ General Director, ${ }^{3,4}$ Resident

${ }^{1,3}$ Department of Ophthalmology, Moscow State University of Medicine and Dentistry named after A.I. Evdokimov, Moscow, Russia

${ }^{2}$ Eye Center "East Sight Recover", Moscow, Russia

${ }^{4}$ The S. Fyodorov Eye Microsurgery Federal State Institution Moscow, Russia

Corresponding Author: SI Anisimov, Professor, Department of Ophthalmology, Moscow State University of Medicine and Dentistry named after A.I. Evdokimov, Moscow, Russia, Phone: 84957896102, e-mail: xen3744@yandex.nu

\section{INTRODUCTION}

At the present time, it is the need to develop the methods for in vivo assessment of biomechanical properties of cornea. Existing methods rely on different physical principles. ${ }^{1-4}$ The most currently used techniques ${ }^{3}$ do not allow us to estimate the distribution of the mechanical properties of cornea on its surface and are integrated. To study keratoconus $(\mathrm{KC})$, we require techniques that allow us to detect local changes in the rigidity of the cornea that may reflect its topographical features. For more complete study of the biomechanics of cornea, it is necessary to estimate not only the distribution of mechanical stress (MS) but also their dependence on its stretchability. We have already published the data that allow us to estimate only the distribution of MS in the cornea in relation of its topography that does not allow us to estimate its elongation. ${ }^{5}$

\section{THE PURPOSE OF THE STUDY}

To develop and evaluate the clinical significance of novel methodology for determining MS and elasticity of the cornea and correlate to its topographic characteristics.

\section{MATERIALS AND METHODS}

A comparative study was conducted in patients that were treated with local crosslinking (Table 1). In every case the corneal zones with high and low rigidity with altered elasticity were identified (reduced or elevated mechanical stress). ${ }^{6}$

Table 1: Clinical characteristics of the patients

\begin{tabular}{ll}
\hline Eyes (patients), $\mathrm{n}$ & $56(72)$ \\
Average age (range) & $32(18-40)$ \\
Female (\%) & 64 \\
Classification of keratoconus, $\mathrm{n}(\%)$ & \\
Suspected keratoconus (patients) & $(1) 2$ \\
Stage 1 & $(27) 31$ \\
Stage 2 & $(22) 33$ \\
Stage 3 & (6) 6 \\
\hline
\end{tabular}


The inclusion criteria of patients in the study were: KC stage I to III (concerning Amsler), ${ }^{7}$ absence of associated ocular pathology.

Exclusion criteria of patients were KC stage IV, related ocular pathology, and previous surgical intervention on the cornea.

The reference group included 15 patients without pathology of the cornea in the age group 18 to 40 years.

Method to study the distribution of MS was carried out as follows: Performing corneal topography using corneal topograph Orbscan II. For further calculation, we used mean power topography's modes and optical pachymetry map. The data in each point of previous topograms are counted according to the Laplace equation,

$$
T=P R / 2 d
$$

To obtain the distribution of tension MS (T), data of corneal curvature $(R)$ were multiplied by the amount of intraocular pressure (IOP) $(\mathrm{P})$ and divided by twice the thickness of the cornea $(\mathrm{d})$. To accelerate the process of calculations, special software calculator Lokolink was used. The resulting topogram we called keratotensotopogram (KTT). It shows the distribution of MS in the cornea, we consider MS also as value characterized by the rigidity of the cornea, i.e., its ability to resist deformation. A normal KTT is shown in Fig. 1 in comparison with the case of KC stage III. To study the distensibility of the cornea, we used vacuum-compression test with the help of vacuum apparatus $^{8}$ - in the field of sclera special hollow cone created vacuum - $40 \mathrm{~mm} \mathrm{Hg}$ (Fig. 2) and in this moment, we registered corneal topogram. Topography before and after the vacuum-compression processed using differential maps and it clearly becomes evident how the curvature of the cornea changed under the influence of IOP. The ratio of stress and strain in the cornea was evaluated at the point corresponding to the visual axis.

\section{RESULTS}

As can be seen in Fig. 1, the distribution of MS in a healthy cornea of the eye and affected with $\mathrm{KC}$ is significantly different. At the initial stage of $\mathrm{KC}$ the areas of reduced rigidity can be clearly identified. In the process of vacuum-compression test, the reaction of the profile of cornea was markedly different in patients with healthy corneas and in patients with KC (Fig. 3). A healthy cornea in all cases increased the curvature in the center. In $\mathrm{KC}$, the cornea is more flat in the center under the IOP elevation (in $100 \%$ cases).
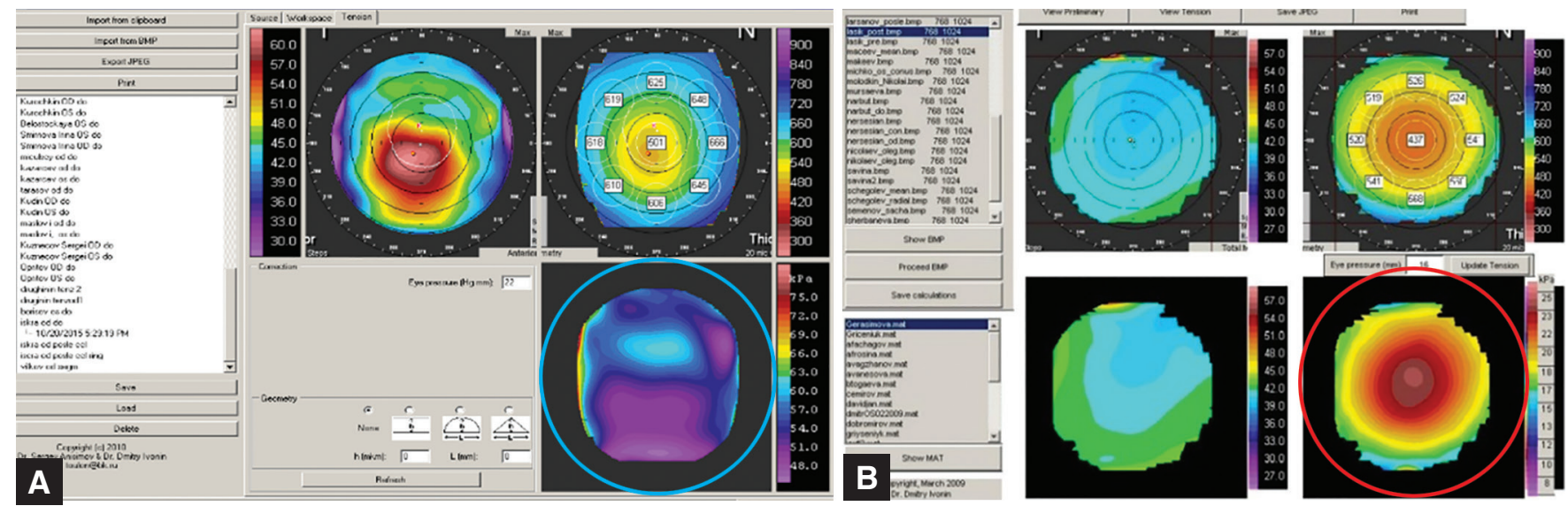

Figs $1 \mathrm{~A}$ and B: Keratotensotopogram in case of keratoconus (II-III stage) in blue ring (rigidity is too low) and normal KTT in red ring

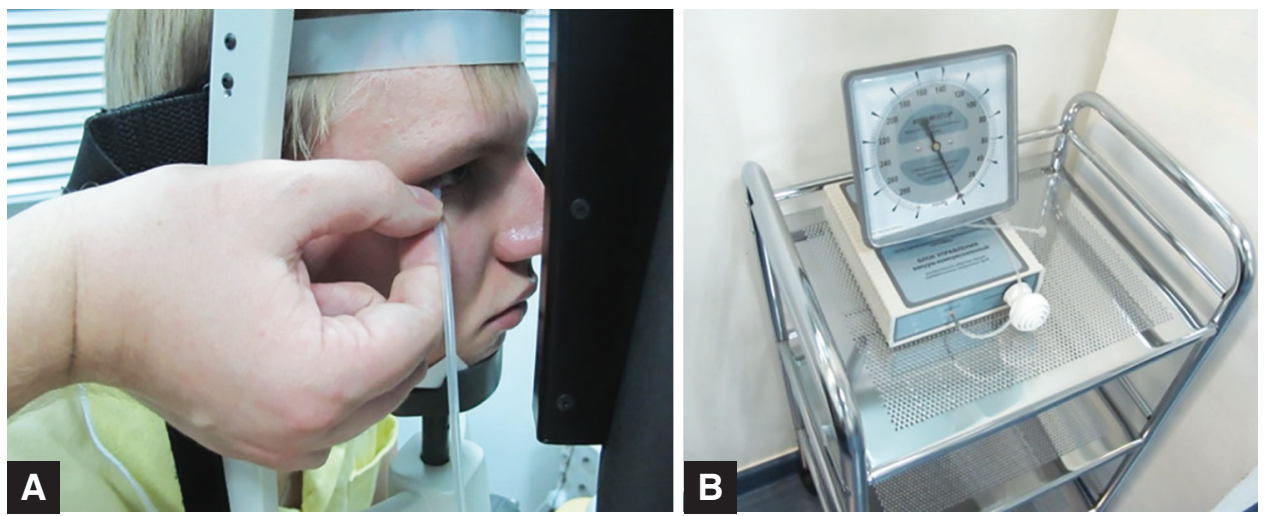

Figs 2A and B: Topographic vacuum-compression test-a method of determining the elasticity of the cornea in keratoconus 


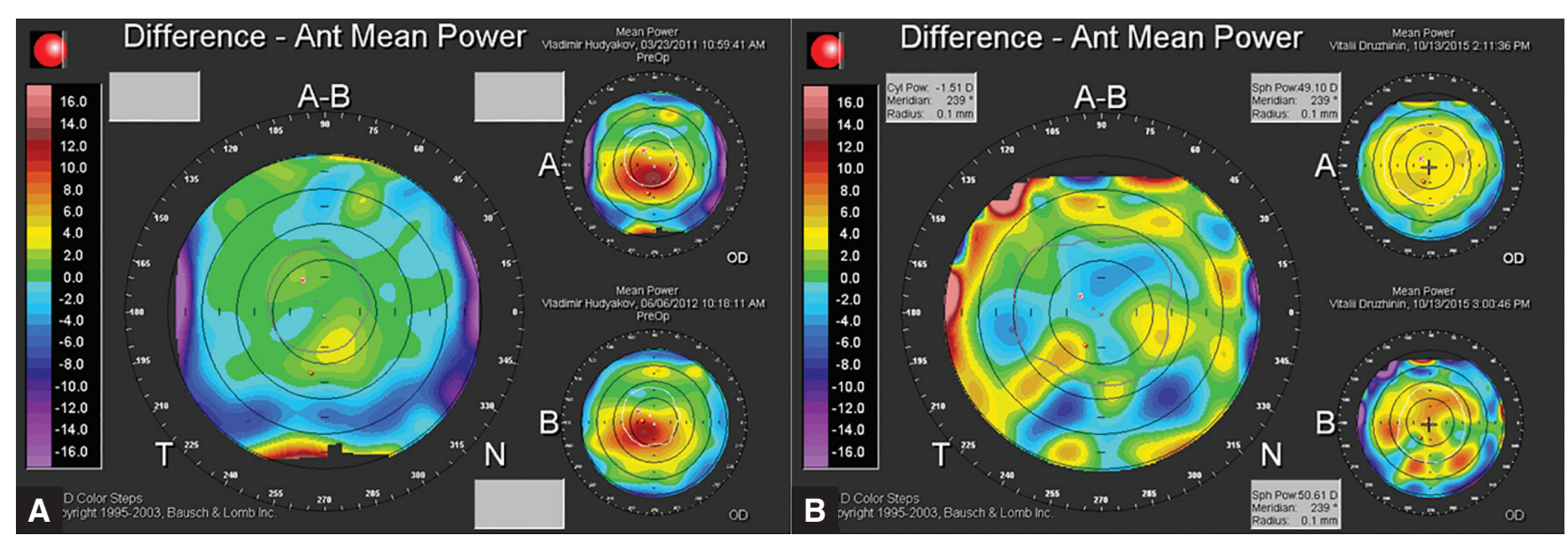

Figs 3A and B: Differential maps with vacuum-compression test. On the left-keratoconus; on the right-normal cornea

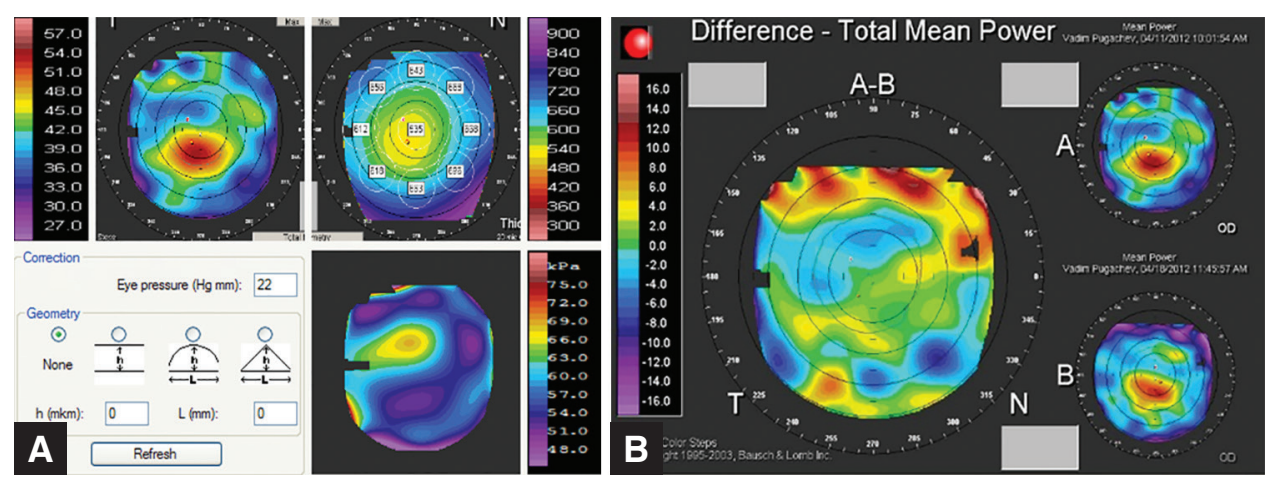

Figs 4A and B: Zone of reduced rigidity of the cornea in keratoconus: (A) Keratotensotopography; (B): differential map after vacuum-compression test

While we checked more advanced stage of $\mathrm{KC}$, the flattening was more noticeable. This indicates the loss of elastic response of the cornea in the region of the apex of the cone. Protrusion of the cornea in KC remains noticeable on the periphery. This area corresponds to the region of reduced rigidity to be detected by KTT (Fig. 4).

We noted that during vacuum compression, the apex of the cone became more flat, and at the same time on periphery, the cornea was expanded. On KTT, the same eye (right) was also determined by the reduction of the rigidity at the periphery of the cornea.

\section{CONCLUSION}

The KTT in eyes with the cornea without pathology differs from KTT in the keratoconic cornea. The KTT and vacuum-compression test can be used in the diagnosis and prediction of biomechanical quality of cornea. Zones of reduced rigidity in the paracentral region contributed hyperextension of the cornea and formation of the apex of KC. Strengthening of weakened areas of cornea, e.g., using corneal segments explains the mechanism of their effect. The proposed diagnostic algorithm allows to determine more precisely areas of lesser corneal rigidity and helps us to provide treatment more accurately particularly in planning localization of UV irradiation for corneal collagen cross-linking.

\section{REFERENCES}

1. Dupps WJ Jr, Wilson SE. Biomechanics and wound healing in the cornea. Exp Eye Res 2006 Oct;83(4):709-720.

2. Roberts C. Biomechanics of the cornea and wavefront-guided laser refractive surgery. J Refract Surg 2002 Sep-Oct;18(5): 589-592.

3. Roberts CJ, Liu J. Corneal biomechanics: from theory to practice. Amsterdam, Netherlands: Kugler Publication; 2016. p. 290.

4. Babara A. Textbook on keratoconus: new insights. London, UK: J.P. Medical Ltd; 2011. p. 248.

5. Anisimov SI, Anisimova SY, Smotrich EA, Zavgorodnyaya TS, Zolotorevsky KA. Keratotensotopography-new diagnostic possibilities for studying the biomechanical properties of the cornea. Ophthalmology 2011;4(8):13-17.

6. Anisimov SI, Pozaritskiy MD, Larionov EV, Anisimova SYu, Kapkova SG, Kurbatova GA, Zolotorevsky KA, Smotritch E.A. First experience of progressive hyperopic shift correction by corneal crosslinking in patients after radial keratotomy. Ophthalmology 2004;4:4-9.

7. Amsler M. La notion du kératocône.Bull Soc Franc Ophtalmol $1951 ; 64: 272-275$.

8. AnisimovSI, AnisimovaSYu, ArutyunyanLL, Chigovanina NP. Changes in biomechanical properties of the eye and optic disk morphometric parameters in response to vacuumcompression stress test. Glaucoma J 2013;29-36. 